\title{
Reference Gene Selection for Quantitative Real-time PCR Normalization in Quercus suber
}

\author{
Liliana Marum*, Andreia Miguel, Cândido P. Ricardo, Célia Miguel* \\ Instituto de Biologia Experimental e Tecnológica (IBET) / Instituto de Tecnologia Química e Biológica-Universidade Nova de Lisboa (ITQB-UNL), Oeiras, Portugal
}

\begin{abstract}
The use of reverse transcription quantitative PCR technology to assess gene expression levels requires an accurate normalization of data in order to avoid misinterpretation of experimental results and erroneous analyses. Despite being the focus of several transcriptomics projects, oaks, and particularly cork oak (Quercus suber), have not been investigated regarding the identification of reference genes suitable for the normalization of real-time quantitative PCR data. In this study, ten candidate reference genes (Act, CACs, EF-1 $\alpha, G A P D H, H i s 3, P s a H$, Sand, PP2A, B-Tub and Ubq) were evaluated to determine the most stable internal reference for quantitative PCR normalization in cork oak. The transcript abundance of these genes was analysed in several tissues of cork oak, including leaves, reproduction cork, and periderm from branches at different developmental stages (1-, 2-, and 3-year old) or collected in different dates (active growth period versus dormancy). The three statistical methods (geNorm, NormFinder, and CV method) used in the evaluation of the most suitable combination of reference genes identified Act and CACs as the most stable candidates when all the samples were analysed together, while B-Tub and PsaH showed the lowest expression stability. However, when different tissues, developmental stages, and collection dates were analysed separately, the reference genes exhibited some variation in their expression levels. In this study, and for the first time, we have identified and validated reference genes in cork oak that can be used for quantification of target gene expression in different tissues and experimental conditions and will be useful as a starting point for gene expression studies in other oaks.
\end{abstract}

Citation: Marum L, Miguel A, Ricardo CP, Miguel C (2012) Reference Gene Selection for Quantitative Real-time PCR Normalization in Quercus suber. PLoS ONE 7(4): e35113. doi:10.1371/journal.pone.0035113

Editor: Shu-Biao Wu, University of New England, Australia

Received October 31, 2011; Accepted March 12, 2012; Published April 18, 2012

Copyright: ( $) 2012$ Marum et al. This is an open-access article distributed under the terms of the Creative Commons Attribution License, which permits unrestricted use, distribution, and reproduction in any medium, provided the original author and source are credited.

Funding: This research was supported by Fundação Ciência e Tecnologia (FCT;http://www.fct.pt), through projects PEst-OE/EQB/LA0004/2011 and PTDC/AGRGPL/098369/2008, and grants BPD/SFRH/47679/2008 (to LM) and BD/SFRH/44474/2008 (to AM). The funders had no role in study design, data collection and analysis, decision to publish, or preparation of the manuscript.

Competing Interests: The authors have declared that no competing interests exist.

*E-mail: marum@itqb.unl.pt (LM); cmiguel@itqb.unl.pt (CM)

\section{Introduction}

The use of reverse transcription quantitative PCR (RT-qPGR) to assess transcript level has been widespread in plant biology. RTqPGR is a sensitive, precise, easy and cost-effective method allowing the detection of low abundant mRNAs and slight variations in gene expression. It has also become the preferred method for the validation of microarray results.

To avoid bias, the use of reliable internal controls for RT-qPGR analysis is essential [1]. Genes required for the maintenance of basic cellular functions, such as actin, $\beta$-tubulin, elongation factor- $1 \alpha$ and $18 S$ rRNA are commonly used as reference genes (RG) or internal controls. In theory, a RG is a gene with a constant level of expression in all cell types and under every experimental condition which may include developmental stages and biotic/abiotic stresses. However, a universal RG does not exist. In fact several studies reported that, according to the experimental conditions and species used, the level of expression of the commonly used RG can often be variable $[2,3,4,5]$, showing that these genes are differentially regulated among experimental conditions and plant species. Furthermore, it has been shown that the conventional use of a single gene for normalization may lead to relatively large errors in a significant proportion of samples [6,7]. Currently, the use of multiple internal control genes is considered as an essential approach for an accurate normalization of data $[5,8,9,10]$. Such an approach relies on the comparison of the mean variation of each gene relative to the mean variation of the other $\mathrm{RG}$ in order to obtain the best normalization factor. Statistical algorithms, such as geNorm [6] and NormFinder [11], were developed to facilitate the evaluation of potential RG expression stability under different experimental conditions. More recently, Hellemans et al. [12] also proposed the Coefficient of variation $(\mathrm{CV})$ method as another powerful indicator of gene stability. Still, geNorm is the only tool that allows us to determine the minimum number of genes to be applied in normalization factor.

While the evaluation of expression stability of potential RG has been addressed under specific conditions for species such as Arabidopsis [2,13,14], barley, wheat and oat [15], rice [16], cotton [17], pea [7], flax [4], medick [18], tomato [19] and tobacco [20], in tree species only a few studies have been reported in poplar, spruce and longan [3,10,21,22,23]. Moreover, there is a general lack of information regarding the suitability of commonly used RG in the RT-qPCR analysis of target genes expressed in recalcitrant hardwood tissues such as wood, bark or cork.

Cork oak (Quercus suber), an evergreen tree characteristic of the Western Mediterranean (Portugal, Spain, Southern France, Italy, North Africa), has a remarkable capacity to produce suberose tissue, the phellem or cork [24], with unique properties that make it an excellent material for industrial applications. Due to the ecological and socio-economic significance of this species, large 
scale transcriptomic projects have been recently launched, targeting specific stress tolerance mechanisms and developmental processes such as cork differentiation $[25,26,27,28]$. Therefore, the need for RT-qPCR approaches to determine, as accurately as possible, the transcript abundance of specific genes is evident. The expression level of target transcripts in cork, as measured by RTqPCR, has already been studied by Soler et al. [29], but a thorough evaluation of the expression stability of RG was not reported.

In order to select the most suitable RG for gene expression quantification by RT-qPGR, we analysed several tissues of cork oak including leaves, reproduction cork and periderm from branches at different developmental stages or collected in alternate seasons. Ten potential RG involved in different biological roles, such as cytoskeleton structure [Actin $($ Act $), \beta$-Tubulin $(\beta$-Tub)], translational elongation [Elongation factor-1alpha $(E F-1 \alpha)$ ], carbohydrate metabolism [glyceraldehyde-3-phophate dehydrogenase (GAPDH)], chromosome organization, biogenesis and nucleosome assembly [Histone 3 (His3)], chloroplast constitution [Photosystem I psaH $(P s a H)]$, vesicle trafficking and endocytosis [SAND family (Sand)], protein modification process [Ubiquitin $(U b q)]$, protein kinase cascade [Serine/threonine protein phosphatase (PP2A)] and intracellular protein transport [Clathrin adaptor complexes medium subunit family $(C A C s)]$, were assessed using several statistical approaches for the normalization of data.

\section{Materials and Methods}

\section{Plant material}

Cork oak leaves, periderm tissues isolated from branches and reproduction cork were used for sampling at these locations. While reproduction cork was harvested from 3 trees growing in Coruche and São Brás de Alportel (Portugal), leaves and branches were collected from a single donor tree at Instituto Superior de Agronomia (Lisboa, Portugal). Young leaves and 1 to 3-year-old branches were collected during the active growth period in May 2010. Three-year-old branches were also collected during the active growth period in April and July 2010 and during the dormancy period in January 2010. Periderm tissues were isolated from branches by peeling off the external bark with a sterile scalpel. Reproduction cork was harvested during the debarking period in July 2009 and 2010. All the harvested tissues were immediately frozen by immersion in liquid nitrogen and stored at $-80^{\circ} \mathrm{C}$ until further use.

\section{Total RNA extraction and purification}

Frozen samples were ground to a fine powder in liquid nitrogen using a mortar and pestle. Total RNA was extracted following a protocol developed for grapevine [30] with minor modifications: (1) all centrifugations were performed at $13200 \mathrm{rpm}$; (2) after the addition of isopropanol the recovered nucleic acids were dissolved in $375 \mu \mathrm{l}$ Tris-EDTA buffer ( $\mathrm{pH} 7.5$ ); (3) total RNA was precipitated with $140 \mu \mathrm{l}$ of $8 \mathrm{M} \mathrm{LiCl}$ overnight at $4^{\circ} \mathrm{C}$ and (4) the final pellet was dissolved in $50 \mu \mathrm{l}$ DEPC-treated water.

To remove any traces of genomic DNA contamination after RNA extraction, two different DNase treatments, DNase I (Qiagen) and TURBO DNase (Ambion), were tested according to the manufacturer's instructions. The RNA samples treated with DNase I were also purified using the RNeasy MinElute Cleanup (Qiagen). The integrity of the RNA samples was assessed by $1 \%$ $(w / v)$ agarose gel electrophoresis with ethidium bromide staining. RNA concentration and the 260/280 and 260/230 nm absorbance ratios were determined using a ND-1000 Spectrophotometer (NanoDrop Technologies Inc., USA). The automated micro- capillary electrophoresis systems currently provide accurate resolution and sensitivity for analysis of RNA quality. By this fact, the first cork oak RNA samples extracted by the methodology described before were analysed by BioAnalyzer 2100 Agilent. However, as a routine procedure, the integrity of the majority of the RNA samples was assessed by $1 \%$ (w/v) agarose gel electrophoresis with ethidium bromide staining.

Absence of genomic DNA contamination was confirmed by performing PCR amplification using total RNA as template and primers designed for amplification of a 1069 bp DNA fragment (F:GGAGGCGTGGAAAGTGTTTA; R:ACTCAAACGCCAACGTAGCA) from the glycerol-3-phosphate acyltransferase 5 gene (GPAT5) coding sequence (GenBank accession number:JN819185).

\section{First-strand cDNA synthesis and quality controls}

cDNA was synthesized from $1.5 \mu \mathrm{g}$ of total RNA using the Transcriptor High Fidelity cDNA Synthesis Kit (Roche) with the anchored-oligo $(\mathrm{dT})_{18}$ primers according to the manufacturer's instructions. To standardize each biological replicate, the products from different cDNA synthesis reactions of the same RNA sample were combined.

In order to ensure reliable results in further steps, the RNA integrity was also checked by amplifying fragments in the $5^{\prime}$ and $3^{\prime}$ regions of SHORT-ROOT (SHR; GenBank accession number:JN819185) cDNA by qPCR. Primers were designed in the $5^{\prime}$ and $3^{\prime}$ regions of the SHR cDNA to amplify fragments of 177 and $193 \mathrm{bp}$, respectively (SHR_5'F: ATGGATACCTTGTTTAGGC; SHR_5'R: GGTTCAGTCGAATTTCGTTC; SHR_3'F: GTAGTGTCTAGAAGAAGAGG; SHR_3'R: GGGTGTAAAGAAGGTACGG). The $3^{\prime} / 5^{\prime}$ ratio was determined according to the following equation: $\left(1+E_{S H R 3^{\circ}}\right)_{S H R 3^{\circ}}^{C q} /\left(1+E_{S H R 5^{\circ}}\right)_{S H R 5^{\circ}}^{C q}[31]$.

\section{Primer design}

Ten candidate RG were evaluated in this study: Act, CACs, EF$1 \alpha$, GAPDH, His3, PsaH, Sand, PP2A, $\beta$-Tub and Ubq. The RG were chosen based on their previous use as internal controls in gene expression studies of hardwood species such as Q.suber, Populus species and V.vinifera, and based on their consistent PCR amplification. RG sequences were obtained from the Fagaceae database, Fagaceae Genome Web (http://www.fagaceae.org/) and from GenBank. Primers were designed using Primer3 software [32] and PCR Primer Stats [33] taking into account the following criteria: annealing temperature of $60^{\circ} \mathrm{C}$, GC content of $42-55 \%$ and primer length of 19-21 bp. The sequence accession numbers, the closest Arabidopsis homolog, as well as the primer sequences and amplicon size, are described in Table 1 . To confirm the specificity of primer annealing the amplicons obtained after PCR amplification were sequenced, with the exception of $A c t$ and $\beta$-Tub already available in Genbank (EU697020 and EE743717, respectively). The amplicon sequences are presented in the supplementary data (Table S1).

\section{qPCR conditions and PCR efficiency}

The experiments were carried out in 96-well plates with a LightCycler 480 (Roche) using SYBR Green I Master (Roche) to monitor the PGR amplification. Reaction mixtures contained $10 \mu \mathrm{l}$ of $2 \times$ SYBR Green I Master, $400 \mathrm{nM}$ of each primer and $1.5 \mu \mathrm{l}$ of cDNA as template, in a total volume of $20 \mu \mathrm{l}$. The following amplification program was used in all $\mathrm{PCR}$ reactions: $95^{\circ} \mathrm{C}$ for $10 \mathrm{~min}, 45$ cycles of $10 \mathrm{~s}$ at $95^{\circ} \mathrm{C}, 10 \mathrm{~s}$ at $60^{\circ} \mathrm{C}$ and $10 \mathrm{~s}$ at $72^{\circ} \mathrm{C}$. The specificity of each amplification reaction was verified by a dissociation curve (melting curve) analysis after the 45 cycles, 
Table 1. Description of the 10 candidate reference genes and primer sequences for RT-qPCR.

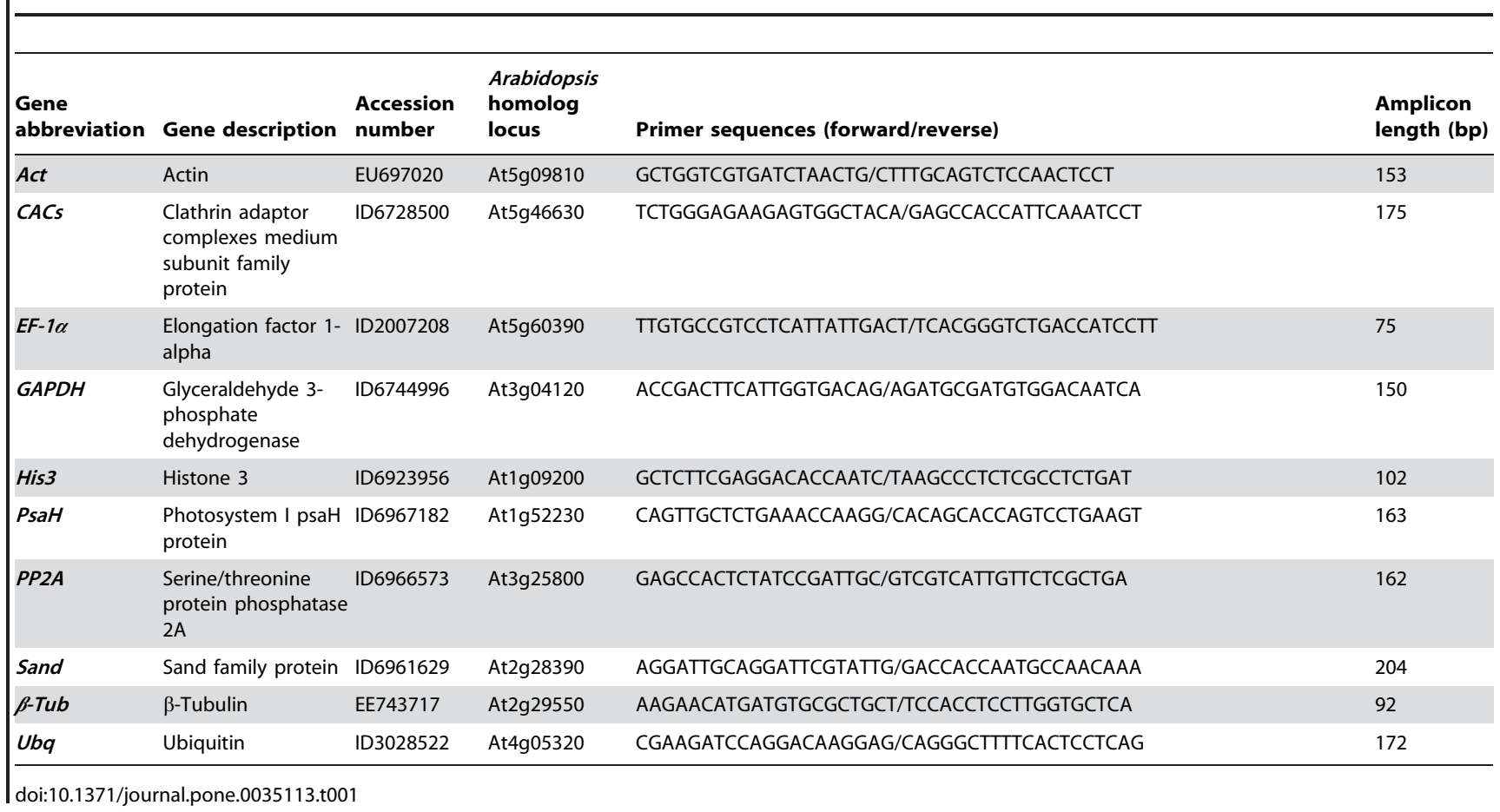

by heating the amplicon from $65^{\circ} \mathrm{C}$ to $97^{\circ} \mathrm{C}$. No-template controls were included for each primer pair.

For all RG studied 2 biological samples were used and the expression levels in each sample were based on 3 technical replicates. Leaf samples were used as calibrator to normalize the values between different plates.

Two different approaches were tested to determine the amplification efficiencies of the RG, using leaves as sample: a standard curve with a three dilution series calculated according to the equation $(1+\mathrm{E})=10^{\text {slope }}$ and the statistical algorithm Real-time PGR Miner. PGR efficiencies (E) for all the other samples were estimated with the Real-time PCR Miner algorithm [34] using the raw fluorescence data as input. Mean efficiency values were obtained for each biological replicate and were used to adjust quantification cycle $(\mathrm{Cq})$ values for subsequent analysis.

\section{Experimental design and data analysis}

Several RT-qPCR experiments were performed to analyse transcript levels in cork oak leaves, cork, periderm from 1, 2 and 3 year-old branches and periderm from 3 year-old dormant branches. For clarity samples corresponding to periderm from 1, 2 and 3 year-old branches together with cork will be referred to as developmental stage set and periderm from 3-year-old branches collected during active growth (May) versus dormancy (January) will be referred to as the seasonal growth sample set. Comparative analyses of all cork oak tissue samples, as well as individual analyses of the several sample types, were also performed.

RG transcript abundance in all the samples was determined by the $\mathrm{Cq}$ value or the number of cycles needed to reach a specific threshold level of detection in the exponential phase of the PCR reaction. Three statistical approaches were used to determine the stability of the candidate RG: geNorm v3.5 [6], NormFinder [11] and $\mathrm{CV}$ method [12]. The Cq values were converted into relative quantities to be used as input data for geNorm and NormFinder (only $\mathrm{Cq}<40$ were used for analysis). The conversion was performed through the formula $Q=E^{\Delta C q}$, where $\mathrm{E}$ is the efficiency of the gene amplification for each primer pair (in each tissue) and $\Delta \mathrm{Cq}$ is the lowest $\mathrm{Cq}$ value as calibrator (which corresponds to the sample with the highest expression) minus the $\mathrm{Cq}$ value of the sample tested. The data obtained from each biological replicate were analyzed in separate. For the CV method the relative quantities were first transformed into normalized relative quantities (formula 15 as in Hellemans et al. [12]) and the CV was calculated using the standard error, through the formulas 17-19 Hellemans, et al. [12]). Finally, the normalization factor (NF) was based on the geometric mean of the best RG selected.

\section{Validation of RG analysis}

One gene of interest putatively coding for a glycerol-3-phosphate acyltransferase 5 (GPAT5) (GenBank accession number: EE743865), was used to validate the selected RG. Primers were designed using Primer3 software [32] (GPAT5_F: GCTAGAGCGGTCTTGACAAAG; GPAT5_R: GACGTCATCAGCTCGCAAAT). The relative expression level of the target gene was determined in periderm tissues from 3-year-old branches collected in April and July 2010. The experimental procedure was the same as used in the selection of RG. For comparative purposes, the relative expression of the target gene was calculated with different normalization factors based on the geometric mean of the two most stable genes [lower $\mathrm{M}$ value, $\mathrm{NF2}(\mathrm{S})$ ] and the two most unstable genes [higher $\mathrm{M}$ value, $\mathrm{NF} 2(\mathrm{U})]$.

\section{Results}

Data normalization using a set of reference genes (RG) is nowadays a current and crucial procedure when analysing the expression levels of target transcripts by RT-qPCR in different tissues or under different conditions. In the present study, the transcript abundance of 10 potential RG was assessed in cork oak by qPCR. A total of 36 cDNA samples including several tissue types and periderm tissues from branches under different 
developmental stages or collected during dormancy versus active growth period, were analysed.

\section{RNA quality}

The assessment of RNA quality encompassed both its purity, characterized by the absence of protein and DNA contamination, and its integrity. All samples were analyzed spectrophotometrically and showed absorbance ratios at 260/280 and 260/230 $\mathrm{nm}$ above 1.8. Total RNA samples were also analysed in agarose gels showing well defined bands corresponding to the rRNA and absence of nucleic acid degradation. To confirm the absence of contaminating genomic DNA, positive and no RT controls were used in GPAT amplification. The DNase I (Qiagen) treatment proved inefficient for the complete removal of genomic DNA from total RNA extracted from cork oak tissues. However, the Turbo DNase (Ambion) proved effective in the removal of DNA contamination, since gene amplification was obtained only from reverse transcribed samples.

The RNA integrity was checked by performing a $3^{\prime}: 5^{\prime}$ assay according to Nolan et al. [31]. This assay gives an indication of the mRNA integrity, since in most cases the RNA degradation starts in the $5^{\prime}$-end region. In general, while a $3^{\prime}: 5^{\prime}$ ratio close to 1 means that the percentage of full-length transcripts in the sample is high, a ratio higher than 5 suggests degradation in the RNA samples. In our study the obtained $3^{\prime}: 5^{\prime}$ ratios were close to 1 with primer efficiencies of 1.8 and 1.9. These results showed that total RNA samples used for RT-qPGR analyses were pure, nondegraded and free of DNA contamination.

\section{qPCR experiments and PCR efficiency}

Specificity of amplification of the several transcripts was supported by the analysis of melting curves and by gel electrophoresis, showing a single PCR amplification product with the expected size for each gene (Fig. S1) and further confirmed by amplicon sequencing. The PCR efficiency (E) of each primer pair was first calculated in cork oak leaves through the standard curve method and then compared with the $\mathrm{E}$ value obtained through the statistical algorithm PCR Miner. According to Czechowski et al. [14] both methods give similar amplification efficiencies. In our study using cork oak leaves, the $\mathrm{E}$ values obtained by both methods were similar (Table S2). However, the standard curve method is time consuming, requiring the production of repeatable and reliable standards [35], with no errors from contamination or sample dilution. Moreover, this method relies on the assumption that the PCR efficiency of each amplicon is constant in all samples, which rarely can be achieved in real experiments [34], strongly influencing the $\mathrm{Cq}$ analysis [36,37]. Therefore, Real-time PCR Miner algorithm [34], using the single raw fluorescence data as an input, was the chosen method to calculate the PGR efficiency for each primer pair in each tissue type (Table 2$)$.

The 10 potential RG tested (Act, $\beta$-Tub, EF-1 $\alpha$, GAPDH, His3, $P s a H$, Sand, Ubq, PP2A and CACs) were successfully amplified in cork oak tissues. The efficiency values $(\mathrm{E})$ were calculated as the mean values obtained from the technical and biological replicates (Table 2), and used to adjust $\mathrm{Cq}$ values for subsequent analysis. Primer efficiencies were higher than 1.9 for all the experiments, except for $U b q$ in cork and for $P s a H$ in periderm from 1 and 2year-old branches and cork, where the values varied between 1.84 and 1.89 (Table 2). Altogether, these results confirm that the selected primers accurately amplify the potential RG.

The calculation of mean $\mathrm{Cq}$ values for the ten $\mathrm{RG}$ in all cDNA samples (Fig. 1) showed a range of variation from 14.8 to 31.0. $G A P D H$ displayed the most abundant transcript level, while $P s a H$ was the less abundant. Based on the interquartile range (25-75\%
Table 2. PCR amplification efficiency of each primer pair.

\begin{tabular}{|c|c|c|c|c|c|c|}
\hline \multirow[b]{2}{*}{ Genes } & \multicolumn{6}{|c|}{ Tissue/stage } \\
\hline & Leaves & $1^{\text {st } B}$ & $2^{\text {nd }} B$ & $3^{r d} B$ & $3^{\text {rd } D B}$ & Cork \\
\hline$A c t$ & $1.93 \pm 0.03$ & $1.96 \pm 0.02$ & $1.94 \pm 0.02$ & $1.90 \pm 0.02$ & $1.91 \pm 0.02$ & $1.94 \pm 0.03$ \\
\hline CACs & $1.93 \pm 0.02$ & $1.97 \pm 0.02$ & $1.94 \pm 0.01$ & $1.94 \pm 0.02$ & $1.97 \pm 0.02$ & $1.95 \pm 0.02$ \\
\hline$E F-1 \alpha$ & $1.92 \pm 0.02$ & $1.95 \pm 0.02$ & $1.92 \pm 0.02$ & $1.96 \pm 0.02$ & $1.96 \pm 0.02$ & $1.93 \pm 0.02$ \\
\hline GAPDH & $1.98 \pm 0.02$ & $1.96 \pm 0.01$ & $1.91 \pm 0.02$ & $1.92 \pm 0.03$ & $1.90 \pm 0.01$ & $1.94 \pm 0.02$ \\
\hline His3 & $1.91 \pm 0.03$ & $1.95 \pm 0.01$ & $1.94 \pm 0.02$ & $1.94 \pm 0.02$ & $1.95 \pm 0.03$ & $1.92 \pm 0.03$ \\
\hline$P P 2 A$ & $1.95 \pm 0.01$ & $1.92 \pm 0.02$ & $1.97 \pm 0.02$ & $1.96 \pm 0.02$ & $1.91 \pm 0.04$ & $1.97 \pm 0.02$ \\
\hline PsaH & $1.93 \pm 0.04$ & $1.89 \pm 0.02$ & $1.89 \pm 0.03$ & $1.92 \pm 0.03$ & $1.94 \pm 0.03$ & $1.84 \pm 0.00$ \\
\hline$U b q$ & $1.94 \pm 0.02$ & $1.91 \pm 0.02$ & $1.92 \pm 0.01$ & $1.93 \pm 0.02$ & $1.94 \pm 0.03$ & $1.86 \pm 0.01$ \\
\hline Sand & $1.92 \pm 0.02$ & $1.92 \pm 0.02$ & $1.95 \pm 0.03$ & $1.91 \pm 0.01$ & $1.91 \pm 0.03$ & $1.93 \pm 0.05$ \\
\hline B-Tub & $1.99 \pm 0.03$ & $1.99 \pm 0.02$ & $1.97 \pm 0.02$ & $1.95 \pm 0.01$ & $1.95 \pm 0.03$ & $1.96 \pm 0.04$ \\
\hline
\end{tabular}

Efficiency values obtained after the amplification of each candidate RG (Act, $B$ Tub, , EF-1 $\alpha$, GAPDH, His3, PsaH, Sand, Ubq, PP2A, CACs) in leaves, periderm from 1,2 and 3 -year-old branches $\left(1^{\text {st }} B, 2^{\text {nd }} B\right.$ and $\left.3^{\text {rd }} B\right)$, periderm from 3-year-old branches in the dormancy period $\left(3^{\text {rd }} \mathrm{DB}\right)$ and cork, estimated with the RealTime PCR Miner algorithm.

doi:10.1371/journal.pone.0035113.t002

percentiles) for $\mathrm{Cq}$ values, the lower $\mathrm{Cq}$ dispersion was observed for Act, CACs, EF-1 1 , followed by Ubq, Sand and PP2A.

\section{Stability analysis}

In order to identify and rank the most suitable RG based on their expression stability, three different statistical approaches, geNorm, NormFinder and CV method, were tested. For all analyses, the $\mathrm{Cq}$ values were transformed into relative quantities using the $\Delta \mathrm{Cq}$ method, and the amplification efficiencies of the RG were calculated by PCR Miner algorithm.

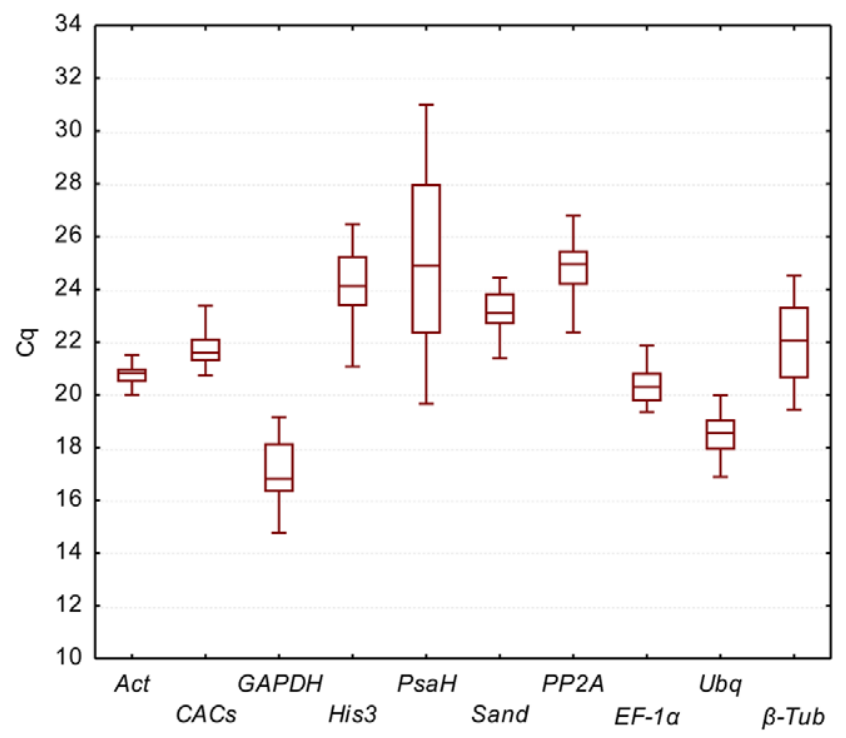

Figure 1. Range of $\mathrm{Cq}$ values of the candidate reference genes obtained for all cDNA samples. Each box corresponding to Act, CACs, EF- $1 \alpha, G A P D H$, His3, Ubq, PsaH, Sand, PP2A and $\beta-T u b$ indicates the $25 \%$ and $75 \%$ percentiles. Whiskers represent the maximum and minimum values. The median is depicted by the line across the box. doi:10.1371/journal.pone.0035113.g001 
When using geNorm algorithm the candidate RG were ranked according to their expression stability measure (M), which represents the average pairwise variation of a particular gene with all other control genes. The stability values are reached after stepwise exclusion of the worst-scoring RG. Considering the data obtained from all samples, Act and CACs were the most stable genes (lowest $\mathrm{M}$ value of 0.462 ), followed by $E F-1 \alpha$ (M value of 0.525) (Fig. 2). Hellemans et al. [12] recommended a stability measure threshold lower than 1 to ensure the most stable genes are selected. In our study six of the genes showed an $\mathrm{M}$ value lower than 1. The highest M value (2.203) was observed for PsaH, the most unstable gene, which can be explained by the lower expression levels observed for this gene in some of the tissues, namely cork and a few periderm samples.

A similar ranking of the tested genes was obtained when expression stability was analysed through the NormFinder algorithm taking into account all cDNA samples (Fig. 2). The three most stable (Act, CACs and EF-1 $\alpha$ ) and the two least stable ( $P s a H$ and His3) candidate genes were the same as identified by geNorm. When using the GV method, Act and CACs were also the best performing genes, but a different ranking of the remaining candidate RG was obtained (Fig. 2).

The analysis of the developmental stage data set by geNorm and NormFinder also revealed a similar ranking of the tested RG, while in the seasonal growth data set analysed by NormFinder and
CV method, the most and less stable genes were similar but the intermediate rank positions differed (Fig. S2). Combining the three statistical approaches in the analysis of the developmental stage and seasonal growth data sets, the $A c t / C A C s / E F-1 \alpha / P P 2 A$ and Act/CACs/GAPDH clusters represented the most stable genes in each set, respectively (Fig. 3).

However, when performing separate analysis of specific tissues, developmental stages or active growth versus dormancy, less uniform results were obtained (Table 3). For instance, in the active growth versus dormancy, CACs/GAPDH, and $E F-1 \alpha /$ Sand can be used as good RG according to geNorm, while NormFinder selected $C A C \mathrm{~s} / G A P D H$ and $U b q$ and $\mathrm{CV}$ method selected CACs and Act as the most adequate for data normalization.

On the other hand, according to Hellemans et al. [12], the mean stability values $(\mathrm{M})$ and the mean $\mathrm{CV}$ for heterogeneous sample panels should be within the $\mathrm{M} \leq 1$ and $\mathrm{CV} \leq 0.5$ ranges. Taking into account all cDNA samples, our results completely match these criteria. The obtained $\mathrm{M}$ values ranged from 0.462 to 0.866, except for the four less stable genes (PP2A, $\beta$-Tub, His3 and $P s a H)$, while $\mathrm{CV}$ values were in the range of $0.198-0.406$. For homogeneous sample panels Hellemans et al. [12], consider a different range, $\mathrm{M} \leq 0.5$ and $\mathrm{CV} \leq 0.25$, for selecting the best $\mathrm{RG}$. The $\mathrm{M}$ and $\mathrm{GV}$ values for the most stable RG selected from the separate analysis of the developmental stages set, seasonal growth
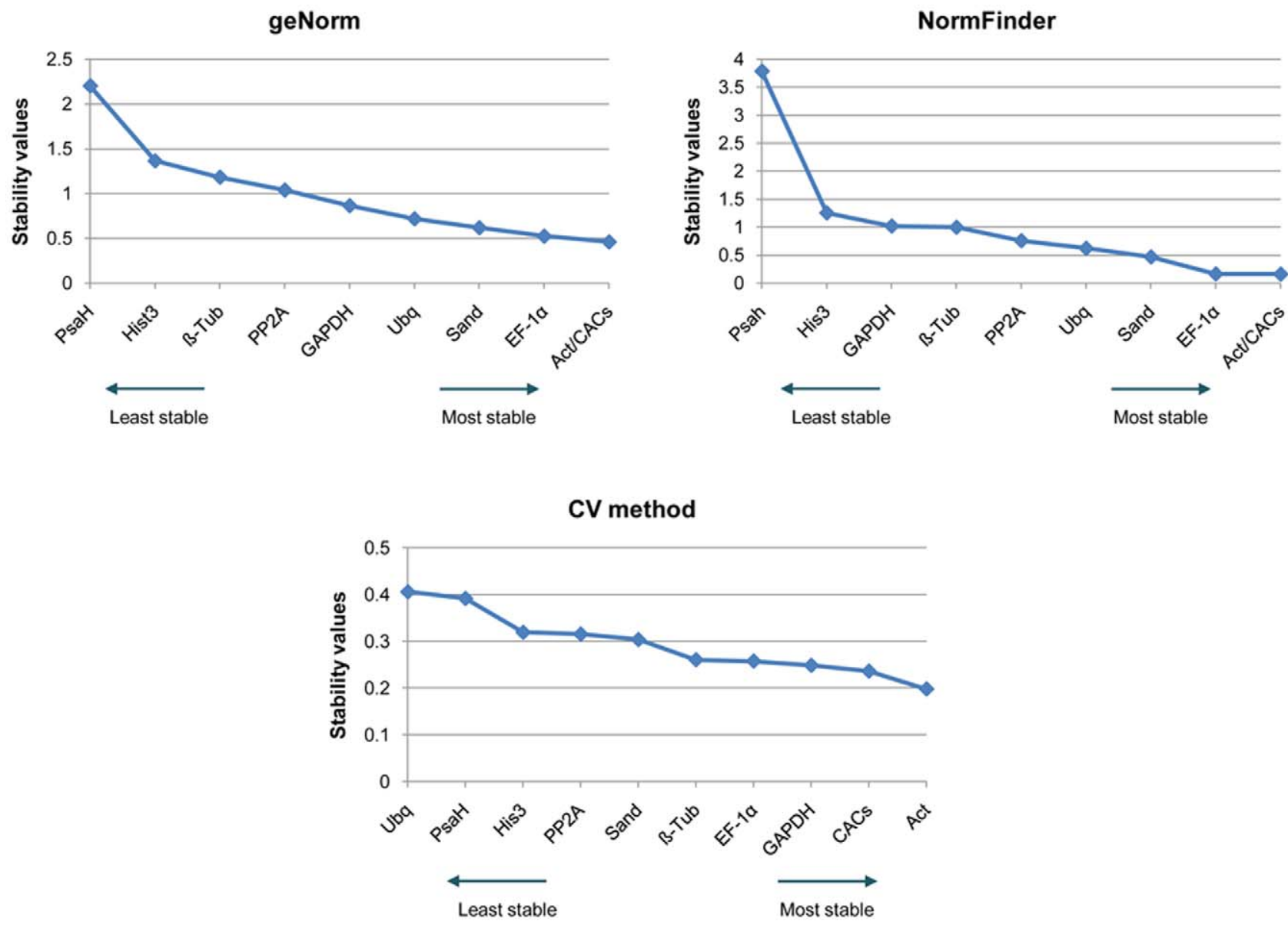

Figure 2. Stability values of candidate reference genes (RG) calculated by different statistical methods using all cDNA samples. Ranking of each RG (Act, CACs, EF-1 $\alpha, G A P D H, H i s 3, U b q, P P 2 A, P s a H$, Sand, $\beta$-Tub), calculated by geNorm, NormFinder and CV method, for all tested samples [leaves, branches $\left(1^{\text {st }}, 2^{\text {nd }}, 3^{\text {rd }}\right.$ active, $3^{\text {rd }}$ dormancy) and cork].

doi:10.1371/journal.pone.0035113.g002 


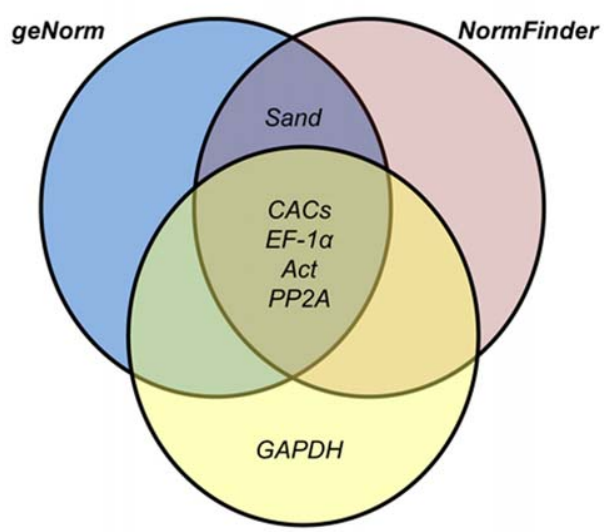

cV

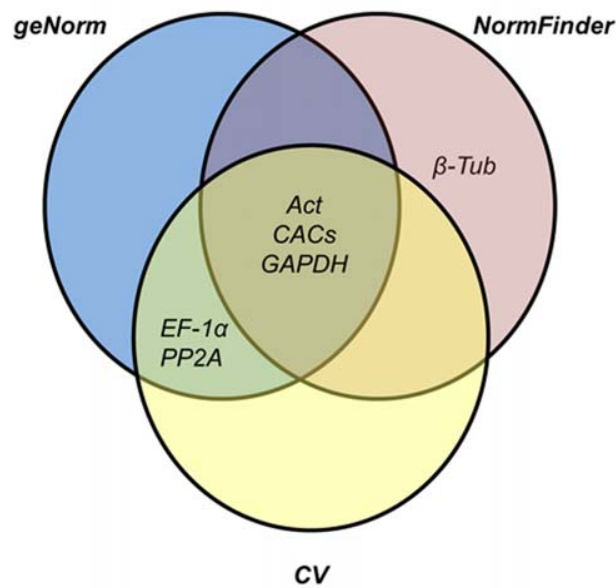

Figure 3. Venn diagram showing the most stable genes identified by the geNorm, NormFinder and CV method. The most stable genes were identified using data from the developmental stage and seasonal growth sample sets. doi:10.1371/journal.pone.0035113.g003

set and the individual analysis of each sample type, also fits the proposed value ranges (Table 3; Fig. S2).

The optimal number of RG used for data normalization was determined through the pairwise variation $\left(\mathrm{V}_{\mathrm{n} / \mathrm{n}+1}\right)$, using the geNorm algorithm [6]. This is calculated between the two sequential normalization factors, $\mathrm{NF}_{\mathrm{n}}$ and $\mathrm{NF}_{\mathrm{n}+1}$ for all the samples under analysis and reveals the effect of adding an $(n+1)^{\text {th }}$ gene, indicating whether the inclusion of an extra reference gene adds stability to the normalization factor. A small variation means no significant effect on the normalization by the addition of another gene. Our study reveals that all $\mathrm{V}_{\mathrm{n} / \mathrm{n}+1}$ values were bellow 0.15 , except when the analysis was performed using the data from all the samples (Fig. 4). Although Vandesompele et al. [6] recommended a cut-off value of 0.15 (bellow which the addition of new gene is not required), this should not be considered as a strict threshold [38] and several subsequent studies have reported higher cut-off values of $\mathrm{V}_{\mathrm{n} / \mathrm{n}+1}[8,39,40]$. Our data shows a small variation between $\mathrm{V}_{2 / 3}(0.169)$ and $\mathrm{V}_{3 / 4}(0.162)$, when all the samples were analysed together, suggesting that the addition of a third gene has no significant effect on the normalization factor.

Table 3. Stability values for the candidate RG in individual sample types.

\begin{tabular}{|c|c|c|c|c|c|c|c|c|c|c|c|}
\hline Method & Tissue/stage & Act & CACs & $E F-1 \alpha$ & $G A P D H$ & His3 & $U b q$ & PsaH & Sand & $P P 2 A$ & B-Tub \\
\hline \multirow[t]{6}{*}{ geNorm } & Leaves & 0.023 & 0.157 & 0.008 & 0.008 & 0.485 & 0.065 & 0.130 & 0.252 & 0.302 & 0.013 \\
\hline & $1^{\mathrm{st} B}$ & 0.030 & 0.087 & 0.009 & 0.194 & 0.138 & 0.068 & 0.002 & 3.38E-04 & 0.272 & 3.38E-04 \\
\hline & $2^{\text {nd }} B$ & 0.098 & 0.276 & 0.022 & 0.255 & 0.145 & 0.059 & 0.843 & 0.209 & 0.432 & 0.022 \\
\hline & $3^{\text {rd }} B$ & 0.167 & 0.050 & 0.218 & 0.050 & 0.288 & 0.415 & 1.086 & 0.590 & 0.080 & 0.686 \\
\hline & $3^{\text {rd }} \mathrm{DB}$ & 0.458 & 0.396 & 0.047 & 0.194 & 1.126 & 0.631 & 1.967 & 0.047 & 1.508 & 0.528 \\
\hline & Cork & 0.548 & 0.608 & 0.679 & 0.472 & 0.233 & 0.308 & 0.214 & 0.086 & 0.052 & 0.052 \\
\hline \multirow[t]{6}{*}{ NormFinder } & Leaves & 0.065 & 0.317 & 0.105 & 0.096 & 0.841 & 0.037 & 0.311 & 0.197 & 0.207 & 0.118 \\
\hline & $1^{\mathrm{st} B}$ & 0.093 & 0.006 & 0.041 & 0.309 & 0.176 & 0.006 & 0.014 & 1.17E-04 & 0.402 & 1.17E-04 \\
\hline & $2^{\text {nd }} B$ & 0.034 & 0.009 & 0.144 & 0.009 & 0.039 & 0.247 & 1.722 & 0.031 & 0.840 & 0.173 \\
\hline & $3^{\text {rd }} B$ & 0.036 & 0.017 & 0.036 & 0.017 & 0.239 & 0.433 & 1.858 & 0.840 & 0.024 & 0.878 \\
\hline & $3^{\text {rd }} \mathrm{DB}$ & 0.467 & 0.571 & 1.190 & 0.946 & 1.153 & 0.116 & 2.616 & 1.152 & 1.609 & 0.163 \\
\hline & Cork & 0.353 & 0.467 & 0.658 & 0.338 & 0.521 & 0.090 & 0.517 & 0.292 & 0.174 & 0.224 \\
\hline \multirow[t]{6}{*}{ CV method } & Leaves & 0.145 & 0.038 & 0.131 & 0.186 & 0.382 & 0.422 & 0.352 & 0.280 & 0.323 & 0.264 \\
\hline & $1^{\mathrm{st} B}$ & 0.182 & 0.253 & 0.193 & 0.115 & 0.289 & 0.273 & 0.249 & 0.219 & 0.319 & 0.201 \\
\hline & $2^{\text {nd }} B$ & 0.141 & 0.182 & 0.159 & 0.163 & 0.129 & 0.216 & 0.440 & 0.170 & 0.320 & 0.216 \\
\hline & $3^{\text {rd } B}$ & 0.167 & 0.055 & 0.211 & 0.257 & 0.127 & 0.377 & 0.444 & 0.365 & 0.207 & 0.352 \\
\hline & $3^{\text {rd }} \mathrm{DB}$ & 0.107 & 0.182 & 0.356 & 0.285 & 0.442 & 0.167 & 0.470 & 0.325 & 0.356 & 0.157 \\
\hline & Cork & 0.345 & 0.377 & 0.395 & 0.299 & 0.298 & 0.325 & 0.485 & 0.348 & 0.227 & 0.207 \\
\hline
\end{tabular}

The stability values for $A c t, \beta-T U B, E F-1 \alpha, G A P D H, H i s 3, P s a H$, Sand, Ubq, PP $2 A, C A C s$ in leaves, periderm from 1,2 and 3 years-old branches and cork $\left(1^{\text {st }} B, 2^{\text {nd }} B, 3^{\text {rd }} B\right)$ or from 3-year-old branches collected in alternate seasons corresponding to active growth versus dormancy $\left(3^{\text {rd }} B, 3^{\text {rd }} D B\right)$ and cork, were calculated by geNorm, NormFinder and CV method. The values in bold refer to the most stable genes.

doi:10.1371/journal.pone.0035113.t003 


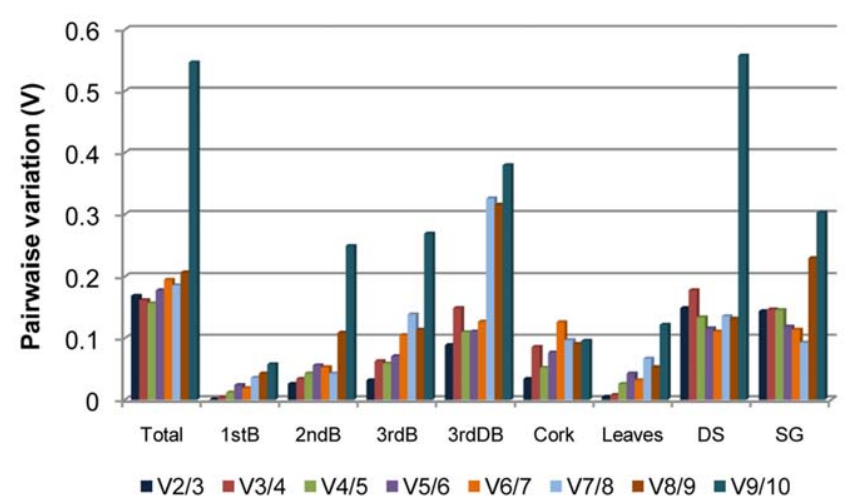

Figure 4. Determination of the optimal number of reference genes for normalization according to geNorm software. Pairwaise variation $\left(V_{n / n+1}\right)$ analysis between the normalization factors $\mathrm{NF}_{\mathrm{n}}$ and $\mathrm{NF}_{\mathrm{n}+1}$, carried out for all the samples (Total), individual samples [leaves, periderm from 1-year-old (1stB), 2-year-old (2ndB) and 3-yearold branches during active growth (3rdB) or dormancy (3rdDB) and cork], developmental stage sample set (DS) and seasonal growth sample set (SG).

doi:10.1371/journal.pone.0035113.g004

\section{RG validation}

The conclusions from the analyses described above were applied to quantify the transcript level of a gene of interest, glycerol-3phosphate acyltransferase 5 (GPAT5). The quantification of transcripts was performed in periderm tissues from 3-year-old branches collected in the spring (April) and summer (July), to validate the RG selected by the different statistical methods. The seasonal variation in GPATs transcript level had been previously evaluated in cork oak by Soler et al. [29] using RT-qPCR.

Different RG combinations were tested in order to assess whether the use of the different normalization factors (NF) obtained by geNorm, NormFinder and CV method had an impact on the transcript quantification results. The several NF were calculated taking into account the stability of the RG as inferred by different statistical methods (geNorm, NormFinder and CV method) and the type of data used for the analysis. Thus, the NF were determined using the two most stable, NF2(S), and the two most unstable genes, NF2(U), identified by geNorm and NormFinder, when analysing (1) data from all the samples [NF2(S) (Act; CACs) and NF2(U) (His3; PsaH)] and (2) data from periderm tissues of 3-year-old branches [NF2(S) (CACs; GAPDH) and NF2(U) $(\beta-T u b ; P s a H)]$ (Fig. 5). The NF were also calculated using the two most stable as well as the two most unstable genes identified by the CV method, when analysing data from all the samples [NF2(S) (Act; CACs) and NF2(U) $(P s a H ; U b q)]$ or data from periderm of 3year-old branches [NF2(S) (CACs; GAPDH) and NF2(U) $(U b q$; $P s a H)]$ (Fig. 5).

When the GPAT expression level was calculated with the NF2(S) obtained by the several statistical methods, a small variation $(<0.5$-fold) between the two seasons (spring and summer) was observed. However, important changes in the relative expression levels ( $\geq 3$ fold) were obtained when the several NF2(U) were used.

The different NF used also enabled us to calculate the average gene specific variation based on the most stable and unstable genes identified from the two type of data used for the analyses. The smallest average gene-specific variation $(22.66 \%)$ was obtained with NF2(S) (Act; CACs), calculated by geNorm and NormFinder, for the data from periderm samples (Fig. 6). The highest genespecific variation, $89 \%$ and $96 \%$, was obtained with the $\mathrm{NF}$ calculated with the most unstable genes from geNorm, NormFin- der and CV method, using data from all the samples and from periderm samples, respectively (Fig. 6).

\section{Discussion}

Gene expression can vary across tissues or cell types as well as developmental and physiological stages. However, the genes commonly referred to as housekeeping function genes (HK) required to maintain basic cellular functions, are expressed in all metabolically active cells or tissues, being critical to the activities that must be carried out for successful completion of cell cycle [41]. Molecular characterization studies have even pointed to characteristic features of HK genes such as a lower degree of conservation of their promoters when compared to those of nonHK genes, and a higher density of SSRs in their 5'-UTRs [42,43]. Due to their role, HK genes have been widely chosen as valuable controls (like RG) in gene expression analyses. Although HK genes continue to be often used, several studies showed that the HK genes are not necessarily expressed at the same level in all tissues $[4,44,45]$. Therefore, it is recognized that a careful choice of RG supported by experimental evidence is essential to obtain a reliable normalization of gene expression data for accurate quantification of transcript levels.

Although some authors still use RG from model plants, several studies have demonstrated that such genes are frequently unstable. For example, the GAPDH gene, involved in basic cellular functions and often assumed to have a uniform expression pattern, is one of the most stable genes in barley, oat and grapevine $[15,30]$ but in $\mathcal{N}$. tabacum it proved to be much less stable [20]. Furthermore, it has been advocated that even for the same type of samples the RG can vary between experiments and laboratories, which can lead to misleading results. Nevertheless, the experimentally induced variations (samples, operator and instruments) can be strongly reduced by implementing a robust methodology.

In this study, the evaluation of ten candidate RG in cork oak samples encompassing different tissues, developmental and seasonal growth stages, was performed following a careful experimental design using several controls for checking RNA quality and integrity and PCR efficiency and specificity. Furthermore, the consistency of the best-scoring RG was tested by several statistical approaches available for this purpose. One of the critical steps addressed in the experimental procedures was to assure the high quality of total RNA isolated from cork oak recalcitrant tissues such as periderm and cork, containing highly suberized cells and high contents of secondary metabolites. This issue was successfully overcome by the use of an optimized RNA extraction protocol and a specific DNAse treatment. The RNA isolation method described here had been previously optimized for cork oak tissues and its effectiveness checked by several available methods including analysis in the Agilent 2100 Bioanalyzer (unpublished results). Although, automated micro-capillary electrophoresis systems currently provide higher resolution and sensitivity for analysis of RNA quality than agarose electrophoresis, the combination of several procedures namely spectrophotometric analysis in the NanoDrop, gel electrophoresis and the $3^{\prime}: 5^{\prime}$-assay used in this work, was adequate for checking the quality of RNA samples isolated through this well established isolation procedure.

As expected, the variation in expression stability of the ten RG tested in our study suggests there is no single RG that can be used for a diversity of cork oak samples. The use of multiple RG that are stable under a given experimental condition was the chosen method for the normalization of RT-qPCR, in agreement with previous reports in species such as Arabidopsis, wheat, barley, oat, 
geNorm/NormFinder_Total

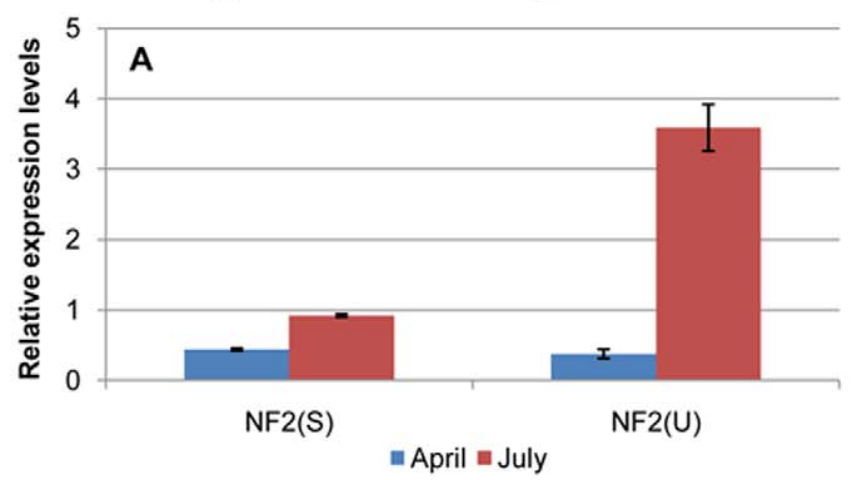

CV method_Total

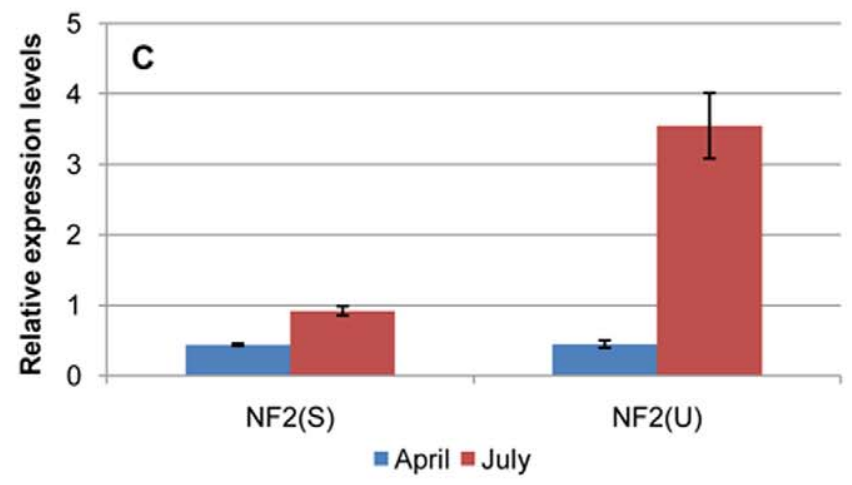

geNorm/NormFinder_3 ${ }^{\text {rd } B}$

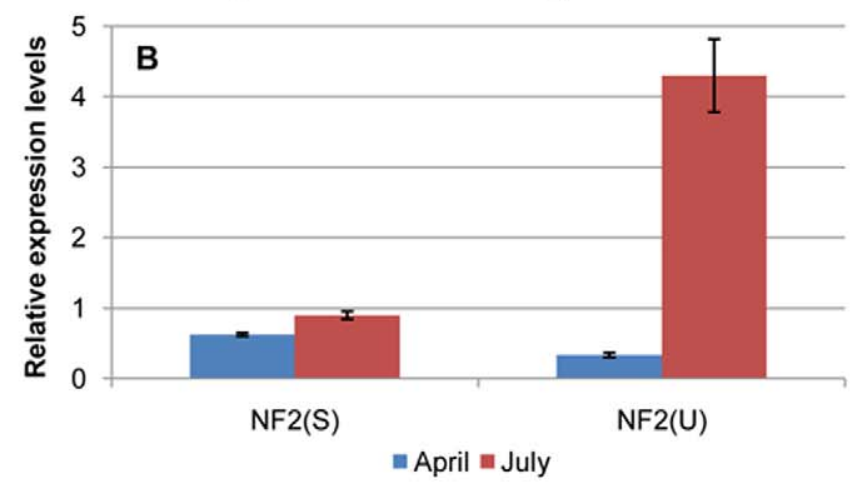

CV method_3 $3^{\text {rd } B}$

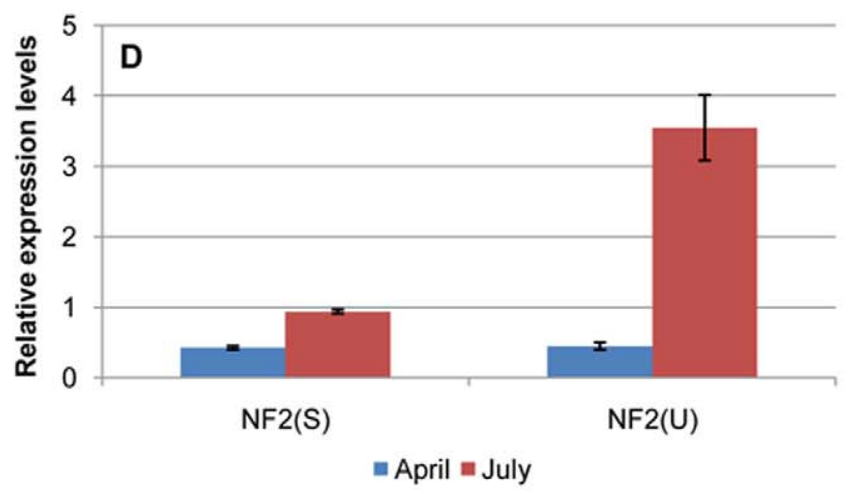

Figure 5. Validation of the reference genes (RG). Relative expression levels of GPAT5 in periderm from 3-year-old branches collected in spring (April) and summer (July). Normalization factors were calculated with RG obtained in the analysis of data from all samples (Total) by geNorm/ NormFinder (A) and CV method (C) or data from periderm of 3-year-old branches ( ${ }^{\text {rd }} B$ ), also in geNorm/NormFinder (B) and CV method (D). The normalization factors were based on the geometric means of the two most stable genes [NF2(S)] and the two most unstable [NF2(U)]. doi:10.1371/journal.pone.0035113.g005

tomato, tobacco, pea, cucumber and poplar [5,7,9,10,19,20], where a similar strategy has proved efficient for the relative quantification of a target gene.

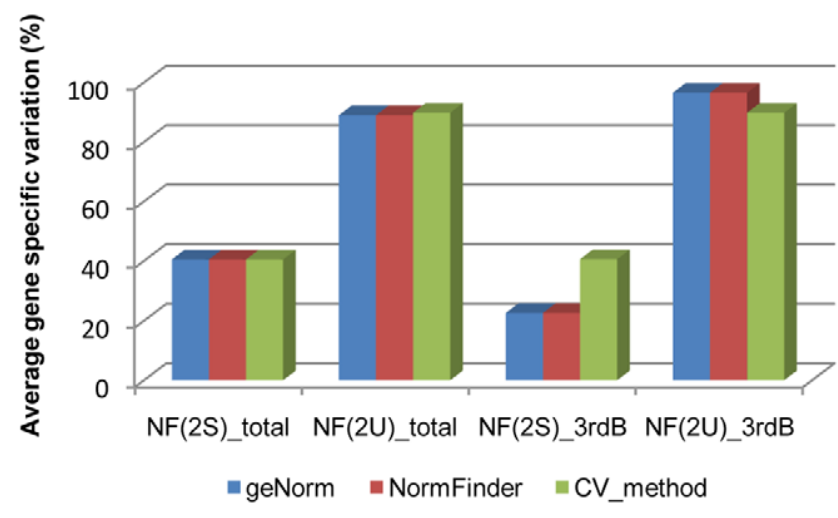

Figure 6. Average GPAT5 gene specific variation. Determination of the coefficient of variation in percentage, for the two most stable, $\mathrm{NF}(2 \mathrm{~S})$, and the two most unstable genes, $\mathrm{NF}(2 \mathrm{U})$. This analysis was performed for the RG selected from all tested samples (total) and from periderm from 3-year-old branches (3rdB), using three statistical methods (geNorm, NormFinder and CV method).

doi:10.1371/journal.pone.0035113.g006
Many of the RG used until now have been selected based on data compiled from microarray databases. However, Czechowski et al. [14] showed that, depending on the specificity of the database used to assess the expression stability, the ranking of the RG genes can change. In order to find adequate RG, several analysis tools have been developed $[6,11,12,46]$ but the question of which procedure is the most suitable remains open. From the different statistical approaches used in this work to analyse gene expression stability, the geNorm and NormFinder algorithms generated a similar RG ranking when considering data from all the samples (heterogeneous sample panel), while the ranking obtained with the GV method was slightly different. Although CACs and Act were within the best-scoring RG by any of the methods, $E F-1 \alpha$ was ranked as one of the most stable genes by geNorm and NormFinder but not by the CV method. Act and EF$1 \alpha$ have been traditionally used as RG namely in $\mathcal{N}$. tabacum, $V$. vinifera and E. ulmoides. The high stability of CACs has also been described in Arabidopsis time-course experiments(Hong et al., 2010), in Cucumis sativus subjected to abiotic stress and exogenously applied growth regulators [5], in different plant structures of Fagopyrum esculentum [9], and in vegetative and reproductive organs of Vaccinium sp [47]. In fact, this gene is involved in a number of essential cellular processes, including membrane trafficking, protein sorting and endocytosis, and it has been identified in Arabidopsis as being among the five most stable genes. On the other hand, $E F-1 \alpha$, one of the genes with a lower $\mathrm{M}$ value in our study, 
was shown to be quite unstable in Arabidopsis [14] and in Salvia miltiorrhiza [48] when geNorm algorithm was used, and also in barley, oat and wheat [9] when three different algorithms were applied. These results confirm that a universal reference gene does not exist, highlighting the need to evaluate commonly used RG for a particular species or condition. When the expression stability was analysed separately for each sample set, the ranking of the RG stability was not uniform. Some of the variation in expression levels may be due to the role of the RG in specific tissues. For example, the role of $\mathrm{PsaH}$ in the chloroplast as a component of photosystem I could explain the low expression levels found for this gene in some of the tested tissues such as cork. On the contrary, $\beta$-Tub was one of the most stable genes in cork. In fact, $\beta$-Tub had already been used as a RG in the quantification of cork transcripts by Soler et al. [29].

Despite the differences we found in the RG ranking when using different statistical approaches, there is a general agreement among the methods for the selection of the most stable genes. The geNorm algorithm is based on the geometric averaging of multiple genes and it has been the most used method $[2,3,4,7,17,20,48]$ due to its simplicity and robustness in the calculation of the NF. The $\mathrm{CV}$ method is another powerful indicator which represents the variation of the normalized quantities across the tested samples while the NormFinder allows estimation, not only of the overall variation of the candidate gene, but also of the variation among sample subgroups. According to Andersen et al. [11], NormFinder is more effective in avoiding the effect of gene co-regulation because it takes into account the intra and inter-group variation. The geNorm software has the advantage of indicating the minimal number of RG required for data normalization through the pairwise variation tool. The use of an increased number of RG in the normalization can improve the reliability of a study, but it is time-consuming and more expensive and thus, a trade-off between the gain in accuracy and the costs and time involved needs to be carefully balanced. In the analysis of our data, the determination of the pairwaise variation of two sequential normalization factors $\left(\mathrm{V}_{\mathrm{n} / \mathrm{n}+1}\right)$ using the geNorm software, indicated that 2 was the minimum number of $\mathrm{RG}$ to be included in the normalization for all the analysed sample sets. The inclusion of a third gene does not add any significant contribute to the calculation of the normalization factor.

Based on previous studies for determination of seasonal variance in transcript abundance of GPAT5 in cork tissues, we chose this gene as a target to validate the RG selected in this study. GPAT5 is involved in suberin biosynthesis, one of the main compounds of cork [49] and it has been identified in a EST collection showing a high and specific expression in the suberin-rich phellem of the cork oak tree [50]. When GPAT5 expression was measured in periderm tissues from 3 year-old cork oak branches no significant variation in transcript abundance was found between April and July, which was in accordance with previous results reported by Soler et al. [29]. The importance of selecting the most stable genes to calculate the NF was evidenced by the huge difference observed in the relative expression levels when NF was calculated with the most stable genes versus the most unstable ones. The genes selected by geNorm and NormFinder, when data from the periderm of 3year-old branches were used, seem to be more reliable to quantify the relative expression levels when comparing to the GV method, as judged by the lowest average gene-specific variation obtained with NF2(S) (Act; CACs).

This study is the first attempt to identify RG in several cork oak tissues. We concluded that Act and CACs were the most stable genes even when considering heterogeneous sample sets, and these were further validated in the transcript quantification of a target gene. These results should be a solid starting point to analyse the expression levels of genes of interest in cork oak or even in other oaks for which large transcriptomics and genomics programs are being developed.

\section{Supporting Information}

Figure S1 Melting curves generated for all amplicons. (TIF)

Figure S2 Stability values of candidate reference genes calculated by different statistical methods using two data sets. The ranking of reference genes (Act, CACs, EF-1 $\alpha$, GAPDH, His3, Ubq, PP2A, PsaH, Sand, $\beta$-Tub) was calculated by geNorm, NormFinder and CV method using the developmental stage and seasonal growth data sets.

(TIF)

Table S1 Amplicon sequences of the 8 candidate reference genes (RG).

(DOG)

Table S2 Amplification efficiencies of the 10 candidate RG measured using the standard curve method.

(DOC)

\section{Acknowledgments}

Dr. Sónia Gonçalves (CEBAL, Portugal) is acknowledged for providing the cork tissue samples used in this work.

\section{Author Contributions}

Conceived and designed the experiments: LM AM CPR CM. Performed the experiments: LM AM. Analyzed the data: LM AM. Contributed reagents/materials/analysis tools: LM AM . Wrote the paper: LM AM.

\section{References}

1. Bustin SA, Benes V, Garson JA, Hellemans J, Huggett J, et al. (2009) The MIQE Guidelines: Minimum Information for Publication of Quantitative Real-Time PCR Experiments. Clinical Chemistry 55: 611-622.

2. Hong SM, Bahn SC, Lyu A, Jung HS, Ahn JH (2010) Identification and Testing of Superior Reference Genes for a Starting Pool of Transcript Normalization in Arabidopsis. Plant \& Cell Physiology 51: 1694-1706.

3. Gutierrez L, Mauriat M, Guénin S, Pelloux J, Lefebvre J-F, et al. (2008) The lack of a systematic validation of reference genes: a serious pitfall undervalued in reverse transcription-polymerase chain reaction (RT-PCR) analysis in plants. Plant Biotechnology Journal 6: 609-618.

4. Huis R, Hawkins S, Neutelings G (2010) Selection of reference genes for quantitative gene expression normalization in flax (Linum usitatissimum L.). BMC Plant Biology 10: 71.

5. Migocka M, Papierniak A (2011) Identification of suitable reference genes for studying gene expression in cucumber plants subjected to abiotic stress and

growth regulators. Molecular Breedingdoi: 10.1007/s11032-11010-1948711030 .

6. Vandesompele J, De Preter K, Pattyn F, Poppe B, Van Roy N, et al. (2002) Accurate normalization of real-time quantitative RT-PCR data by geometric averaging of multiple internal control genes. Genome Biology 3: 34.31-34.11.

7. Die J, Román B, Nadal S, González-Verdejo C (2010) Evaluation of candidate reference genes for expression studies in Pisum sativum under different experimental conditions. Planta 232: 145-153.

8. Maroufi A, Bockstaele EV, Loose MD (2010) Validation of reference genes for gene expression analysis in chicory (Cichorium intybus) using quantitative real-time PCR. BMC Plant Biology 11: 15.

9. Demidenko NV, Logacheva MD, Penin AA (2011) Selection and Validation of Reference Genes for Quantitative Real-Time PCR in Buckwheat (Fagopyrum esculentum) Based on Transcriptome Sequence Data. Plos One 6: e19434. 
10. Xu M, Zhang B, Su X, Zhang S, Huang M (2011) Reference gene selection for quantitative real-time polymerase chain reaction in Populus. Analytical Biochemistry 408: 337-339.

11. Andersen CL, Jensen JL, Ørntoft TF (2004) Normalization of Real-Time Quantitative Reverse Transcription-PCR Data: A Model-Based Variance Estimation Approach to Identify Genes Suited for Normalization, Applied to Bladder and Colon Cancer Data Sets. Cancer Research 64: 5245-5250.

12. Hellemans J, Mortier G, De Paepe A, Speleman F, Vandesompele J (2007) qBase relative quantification framework and software for management and automated analysis of real-time quantitative PCR data. Genome Biology 8: R19.

13. Remans T, Smeets K, Opdenakker K, Mathijsen D, Vangronsveld J, et al. (2008) Normalisation of real-time RT-PCR gene expression measurements in Arabidopsis thaliana exposed to increased metal concentrations. Planta 227: 1343-1349.

14. Czechowski T, Stitt M, Altmann T, Udvardi MK, Scheible W-R (2005) Genome-Wide Identification and Testing of Superior Reference Genes for Transcript Normalization in Arabidopsis. Plant Physiology 139: 5-17.

15. Jarošová J, Kundu J (2010) Validation of reference genes as internal control for studying viral infections in cereals by quantitative real-time RT-PCR. BMC Plant Biology 10: 146.

16. Li O-F, Sun S, Yuan D-Y, Yu H-X, Gu M-H, et al, (2010) Validation of Candidate Reference Genes for the Accurate Normalization of Real-Time Quantitative RT-PCR Data in Rice During Seed Development. Plant Molecular Biology Reporter 28: 49-57.

17. Artico S, Nardeli S, Brilhante O, Grossi-de-Sa M, Alves-Ferreira M (2010) Identification and evaluation of new reference genes in Gossypium hirsutum for accurate normalization of real-time quantitative RT-PCR data. BMC Plant Biology 10: 49.

18. Kakar K, Wandrey M, Czechowski T, Gaertner T, Scheible W-R, et al. (2008) A community resource for high-throughput quantitative RT-PCR analysis of transcription factor gene expression in Medicago truncatula. Plant Methods 4: 18.

19. Expósito-Rodríguez M, Borges AA, Borges-Pérez A, Pérez JA (2008) Selection of internal control genes for quantitative real-time RT-PCR studies during tomato development process. BMC Plant Biology 8.

20. Schmidt G, Delaney S (2010) Stable internal reference genes for normalization of real-time RT-PCR in tobacco (Nicotiana tabacum) during development and abiotic stress. Molecular Genetics and Genomics 283: 233-241.

21. Phillips M, D'Auria J, Luck K, Gershenzon J (2009) Evaluation of Candidate Reference Genes for Real-Time Quantitative PCR of Plant Samples Using Purified cDNA as Template. Plant Molecular Biology Reporter 27: 407-416.

22. Brunner A, Yakovlev I, Strauss S (2004) Validating internal controls for quantitative plant gene expression studies. BMC Plant Biology 4: 14

23. Lin YL, Lai ZX (2010) Reference gene selection for qPCR analysis during somatic embryogenesis in longan tree. Plant Science 178: 359-365.

24. Silva SP, Sabino MA, Fernandes EM, Correlo VM, Boesel LF, et al. (2005) Cork: properties, capabilities and applications. International Materials Reviews 50: $345-365$.

25. Marum L, Miguel A, Ricardo PC, Miguel C (2011) Identification of GPAT acyltransferases in cork oak. Arraial d'Ajuda, Bahia, Brazil. BMC Proceedings. P69 p.

26. Miguel A, Ricardo PC, Jones B, Miguel C (2011) Identification of a putative molecular regulator of cork cambium. Arraial d'Ajuda, Bahia, Brazil. BMC Proceedings. P70 p.

27. Ramos M, Rocheta M, Carvalho L, Graça J, Morais-Cecilio L (2011) Expression analysis of DNA methyltransferase and co-repressor genes in Quercus suber phellogen: an attempt to correlate with cork quality. Arraial d'Ajuda, Bahia, Brazil. BMC Proceedings. P169 p.

28. Paiva JA, Fevereiro P, Marques P, Rodrigues JC, Provost GL, et al. (2011) Deciphering cork formation in Quercus suber. Arraial d'Ajuda, Bahia, Brazil. BMC Proceedings. P172 p.
29. Soler M, Serra O, Molinas M, García-Berthou E, Caritat A, et al. (2008) Seasonal variation in transcript abundance in cork tissue analyzed by real time RT-PCR. Tree Physiology 28: 743-751.

30. Reid K, Olsson N, Schlosser J, Peng F, Lund S (2006) An optimized grapevine RNA isolation procedure and statistical determination of reference genes for real-time RT-PCR during berry development. BMC Plant Biology 6: 27.

31. Nolan T, Hands RE, Bustin SA (2006) Quantification of mRNA using real-time RT-PCR. Nature Protocols 1: 1559-1582.

32. Rozen S, Skaletsky H, eds. Primer3 on the WWW for general users and for biologist programmers. Totowa, NJ: Humana Press. pp 365-386.

33. Stothard P (2000) The Sequence Manipulation Suite: JavaScript programs for analyzing and formatting protein and DNA sequences. Biotechniques 28: $1102-1104$.

34. Zhao S, Fernald RD (2005) Comprehensive algorithm for quantitative real-time polymerase chain reaction. J Comput Biol 12: 1047-1064.

35. Pfaffl MW (2001) A new mathematical model for relative quantification in realtimt RT-PCR. Nucleic Acids Research 29: e45.

36. Ramakers C, Ruijtera JM, Depreza RHL, Moorman AFM (2003) Assumptionfree analysis of quantitative real-time polymerase chain reaction (PCR) data. Neuroscience Letters 339: 62-66.

37. Kamphuis W, Schneemann A, Beek LMV, Smit AB, Hoyng PFJ, et al. (2001) Prostanoid receptor gene expression profile in human trabecular meshwork: a quantitative real-time PCR approach. Investigative Ophthalmology \& Visual Science 42: 3209-3215.

38. - geNorm software manual (2007) Available: http://medgen.ugent.be/ $\sim$ jvdesomp/genorm/-. Accessed 2012 Mar 28.

39. Kuijk EW, Puy Ld, Tol HTv, Haagsman HP, Colenbrander B, et al. (2007) Validation of reference genes for quantitative RT-PCR studies in porcine oocytes and preimplantation embryos. BMC Developmental Biology 7: 58.

40. Silveira ÉD, Alves-Ferreira M, Guimarães LA, Silva FRd, Carneiro VTdC (2009) Selection of reference genes for quantitative real-time PCR expression studies in the apomictic and sexual grass Brachiaria brizantha. BMC Plant Biology 9: 84 .

41. Warrigton JA, Nair A, Mahadevappa M, Tsyganskaya M (2000) Comparison of human adult and fetal expression and identification of 535 housekeeping/ maintenance genes. Physiological Genomics 2: 143-147.

42. Lawson MJ, Zhang L (2008) Housekeeping and tissue-specific genes differ in simple sequence repeats in the 5'-UTR region. Gene 407: 54-62.

43. Farré D, Bellora N, Mularoni L, Messeguer X, Albà MM (2007) Housekeeping genes tend to show reduced upstream sequence conservation. Genome Biology 8: R140.

44. Kouadjo KE, Nishida Y, Cadrin-Girard JF, Yoshioka M, St-Amand J (2007) Housekeeping and tissue-specific genes in mouse tissues. BMC Genomics 8: 127.

45. Zhang L, Li W-H (2004) Mammalian Housekeeping Genes Evolve More Slowly than Tissue-Specific Genes. Molecular Biology and Evolution 21: 236-239.

46. Pfaffl MW, Tichopad A, Proomet C, Neuvians TP (2004) Determination of stable housekeeping genes, differentially regulated target genes and sample integrity: BestKeeper - Excel-based tool using pair-wise correlations. Biotechnol Lett 26: 509-515.

47. Vashisth T, Johnson LK, Malladi A (2011) An efficient RNA isolation procedure and identification of reference genes for normalization of gene expression in blueberry. Plant Cell Reportdoi: 10.1007/s00299-00011-01121-z.

48. Yang Y, Hou S, Cui G, Chen S, Wei J, et al. (2010) Characterization of reference genes for quantitative real-time PCR analysis in various tissues of Salvia miltiorrhiza. Molecular Biology Reports 37: 507-513.

49. Beisson F, Li Y, Bonaventure G, Pollard M, Ohlrogge JB (2007) The acyltransferase GPAT5 is required for the synthesis of suberin in seed coat and root of Arabidopsis. The Plant Cell Vol 19: 351-368.

50. Soler M, Serra O, Molinas M, Huguet G, Fluch S, et al. (2007) A Genomic Approach to Suberin Biosynthesis and Cork Differentiation. Plant Physiology 144: 419-431. 